\title{
A Sallen Key All Pass Filter for Noise Reduction in Visible Light Communication Systems
}

\author{
Sharanbasappa Shetkar, Baswaraj Gadgay
}

\begin{abstract}
Visible-light communication (VLC) uses a solid-state illumination device for generating high speed communication links. VLC is used throughout world for eliminating the shortage of wireless spectrum resources. The VLC is generally an Optical Wireless Communication (OWC) method and this uses the Light Emitting Diode (LED) for data transmission through the optical wireless channel. The data transmission though the VLC is affected by ambient light interference due to the environment. In this paper, an efficient filter is designed to eliminate the ambient light noises from the received signal. The filter used in the VLC system is Sallen key All Pass Filter (APF) and the red LED and Photo detector (PD) is used in the transmitter and receiver respectively. The Sallen key APF contains three different filter structures such as low pass, band pass and high pass filter. The Sallen APF uses the filter process with respect to the noise. This proposed system is implemented in two different tools namely MATLAB and Electric $V L S I$. The performance of the proposed system is analyzed in terms of Bit Error Rate (BER), area, power consumption and data rate.
\end{abstract}

Index Terms: Visible-light Communication, Sallen Key All Pass Filter, Bit Error Rate, Data Rate, Light Emitting Diode.

\section{INTRODUCTION}

Nowadays, wireless data traffic grows exponentially due to the huge population of smart mobile devices. This leads to creates the shortage of the Radio Frequency (RF) spectrum and also it creates the RF spectrum to be more precious and expensive [1]. Moreover, the RF-based systems are not allowed in some places such as airplanes and hospitals [2]. The VLC is used instead of the RF bands for short-range communication. VLC is considered as modern Optical Wireless Communication (OWC) technologies that depend on the visible light region (i.e., 375-780 $\mathrm{nm}$ in wavelength) of electromagnetic spectrum. This region is considered as a medium for data transmission between the users [3]. In the communication system design, the lighting constraint is considered for achieving the communication requirements of various VLC lighting environments [4]. The VLC uses the Light Emitting Diode (LED) for green data communication. This LED is utilized as an alternative for the fluorescent

Revised Manuscript Received on October 30, 2019

* Correspondence Author

Sharanbasappa Shetkar*, Electronics and Communication Engineering, Lingaraj Appa Engineering College, Bidar, India.

Dr. Baswaraj Gadgay, Electronics and Communication Engineering, VTU-RO Kalaburagi, India.

(C) The Authors. Published by Blue Eyes Intelligence Engineering and Sciences Publication (BEIESP). This is an open access article under the CC BY-NC-ND license (http://creativecommons.org/licenses/by-nc-nd/4.0/) lamps and incandescent light bulbs due to its long lifetime, compact size, huge electric-to-optic conversion efficiency, etc. The VLC has various advantages such as easy to implement, high transmission speed, high data rates, dual usage (illumination and data transmission), harmlessness for human and inherent data security [5], [6].

In VLC, generally the LED is act as a transmitter and photodiode is operated as a receiver. Here the photodiode has a higher linearity range and modulation bandwidth than the LED [7]. Nevertheless, the VLC is controlled by the LED's bandwidth and also by some approaches like complex modulation and equalization which is used for enhancing the data rate [8]. The LED based VLC is enabled in several applications such as illumination, broadcasting, sensing networks, intelligent transportation systems, etc [9]. In VLC, the capability of data transmission is obtained by modulating the data into the light's instantaneous power [10]. The VLC is generally utilized in two different scenarios such as outdoor and indoor. In the outdoor scenario, the VLC is used for the intelligent transportation system [11]. In the indoor communications, the LEDs are referred to as access points which are used to provide the transmission within small range of cell that is denoted as attocell [12]. The ambient light interference is considered as main issue in the design of VLC. The noise sources of indoor is fluorescent lights/incandescent lights and also for outdoor is sun light that occupies broad range of wavelength [13] [14]. The type of filters used in the existing researches are color filter [15], audio filter [16], band pass filter [17], etc. The major contributions of this research are stated as follows:

- Due to high sensing capacity the red LED is chosen among various LED types to perform the data transmission among the VLC system.

- Sallen key APF is used in the VLC system to minimize the ambient light interference from the various sources like incandescent, fluorescent lamps, skylight and sunlight.

\section{LITERATURE SURVEY}

Che, F., Wu, L., Hussain, B., Li, X. and Yue, C.P [18] presented the VLC transmitter by using VLC modulator, IEEE 802.15.7 PHY-I baseband digital signal processing unit, LED array and dc-dc boost power converter. Here, the white LEDs are used as broadcasters or beacons to transmit the data. In higher data rate VLC communications, the IEEE 802.15.7 standard is used instead of the nonstandard-based modulation at less distance.

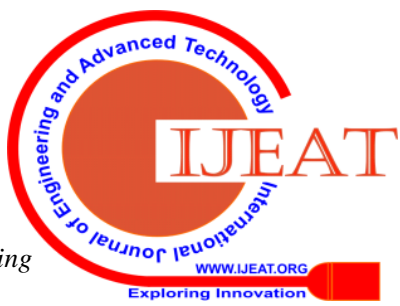


This VLC system is implemented in the process of .35- $\mu \mathrm{m}$ CMOS. The transmission bit efficiency of the VLC system is $5 \mathrm{~nJ} /$ bit at the distance of $2 \mathrm{~m}$. In the brightness level of VLC system, the error will occur when the modulation frequency is at $25 \mathrm{kHZ}$.

Li, X., Hussain, B., Wang, L., Jiang, J. and Yue, C.P [19] introduced the energy efficient VLC receiver system-on-a-chip (SoC). This VLC system uses the post-equalization techniques and Ambient Light Rejection (ALR) in light fidelity applications. The phosphorescent white LEDs are used in the VLC system. The ambient light causes the potential DC differences in the VLC communication system which is eliminated by using the ALR unit. The bandwidth created by white LED (i.e., under 3 $\mathrm{MHz}$ ) is compensated by using the two-stage continuous-time linear equalizer. This VLC SoC system is developed in a 0.18 $\mu \mathrm{m}$ CMOS process. The performance of the system may be varied due to the misalignment of the transmitter and receiver.

Mahfouz, N.E., Fayed, H.A., El Aziz, A.A. and Aly, M.H [20] used the16 LED array of LED arrangement design to create the indoor VLC system. The system uniformity is improved by increasing the number of transmitters. The main objective of this work is to reduce SNR oscillations and power without increasing LED arrays. Here, the phosphorescent white LED is used as the light source of VLC system. In this VLC system, the problems due to the optical noise are minimized by using the optical filter. Besides, the optical concentrator is used in the receiver for improving the signal to noise ratio. This VLC system performance is analyzed under various levels of half power angle. The significant amount of ISI is generated during the increment in power.Kadirvelu, S. and Baba, V [21] introduced the indoor wireless communication using LED as both transmitter and receiver. In this VLC system, the digital signal is transmitted using red-to-red LED. The LED used in this VLC system is modulated by using the on-off keying and the line of sight is considered as a channel model among the transmitter and receiver. Then ambient light conditions are used to evaluate the performance of the VLC system. Here, the RC high pass filter is used at the receiver section to avoid the noise from the ambient light. Because generally the ambient light noise from the environment can reduce the performance of the VLC communications. The filter structure used in this work fails to consider the noise which is in the bands of low pass and band pass. Gao, Y., Li, L. and Mok, P.K [22] presented the VLC system with the utilization of digitalized power stage and controller to create better communication. Here both the VLC application and illumination are achieved by using the inductor-less LED driver with the features of $8-\mathrm{W}, 89.2 \%$ efficiency. Here two turn on speed enhancement techniques are used to maximize the peak data rate. This VLC system is implemented based on $0.35-\mu \mathrm{m}$ technology. The flicker present in the output light is eliminated by using the Low Pass Filter (LPF). Hence, the low frequency flicker is reduced to below $10 \%$. In this work, the redundancy can be included in the coding to preform error correction which leads to high-quality communication with an effective BER. But, in that case the maximum data rate received by the receiver is decreased.

\section{PROPOSED SYSTEM}

The ambient light noise is added during the data transmission between the LED and PD through the optical wireless channel. Some source of ambient light noise is skylight, sunlight, incandescent, fluorescent lamps, etc. The addition of noise from the environment degrades the performance of VLC. So in this proposed system, the Sallen-key all pass filter is used for eliminating the ambient light noise from the environment. The Sallen key is used because of its less cost and less complexity. Moreover, the component requirements used in this filter is less as well as the resistors and capacitors are considered to be equal. The overall view of the proposed system is shown in the following Fig. 1.

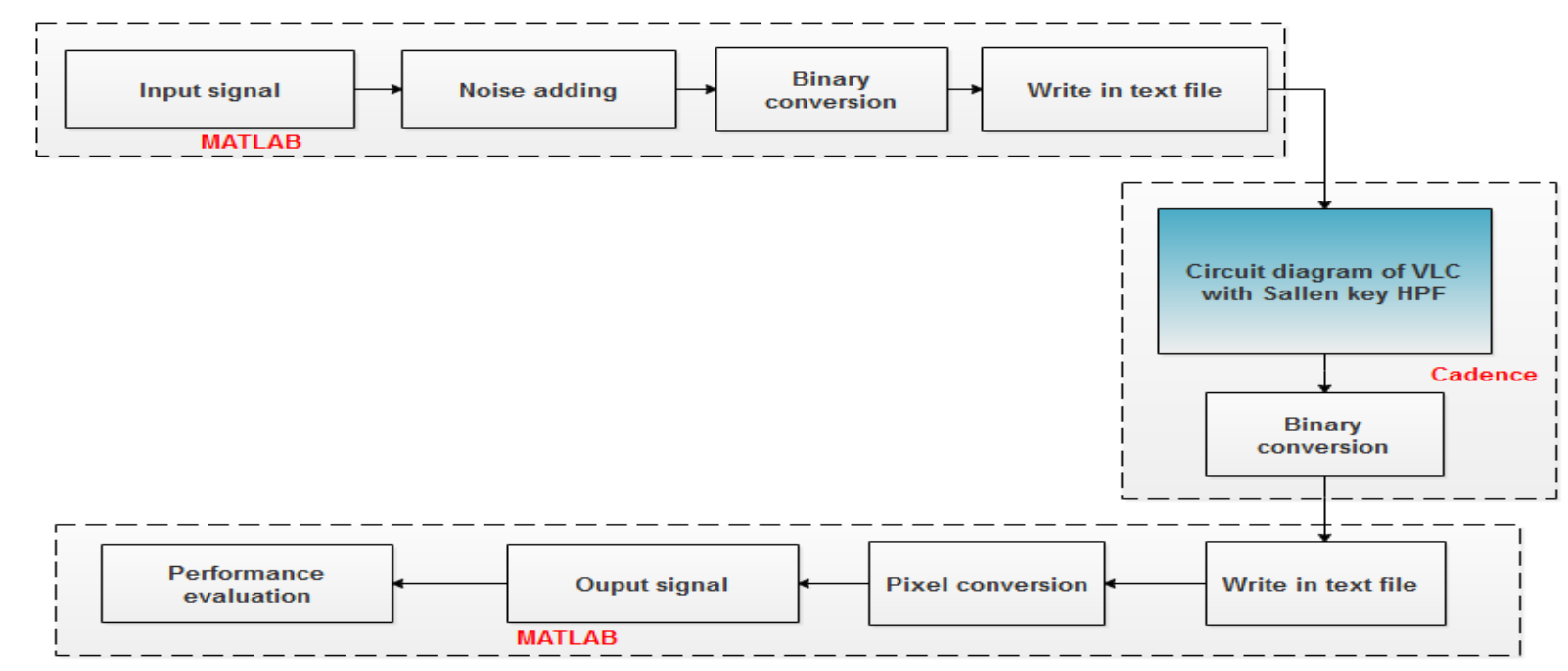

Fig. 1. Block diagram of the proposed system 
The overall process of the proposed system contains six steps: 1. Input signal acquisition, 2. Convert input signal into binary form, 3. Data transmission using LED, 4. Data receiving by using PD, 5. Filtering using Sallen-key APF 6. Convert binary form into the signal. Initially, the input signals are acquired from the MATLAB software. Then these signals are converted in to binary form for performing the data transmission. The binary form values from the MATLAB is saved in the file extension of .txt. Additionally, this txt file is used in the electric VLSI circuit for VLC communication. In that VLC communication the data is transferred between red LED to PD. Here, the Sallen key APF is designed in the receiver section by using the electric VLSI to filter the noise occurred due to the sunlight, fluorescent lamps, etc. This Sallen key APF performs three different filtering process such as low pass, band pass and high pass. The filter structure is operated according to the noise levels. Finally, the received binary form signals are again given as input to the MATLAB and it is again converted into the signal from a binary form. Then the equivalent circuit diagram of the VLC using Sallen-key APF is shown in Fig. 2.

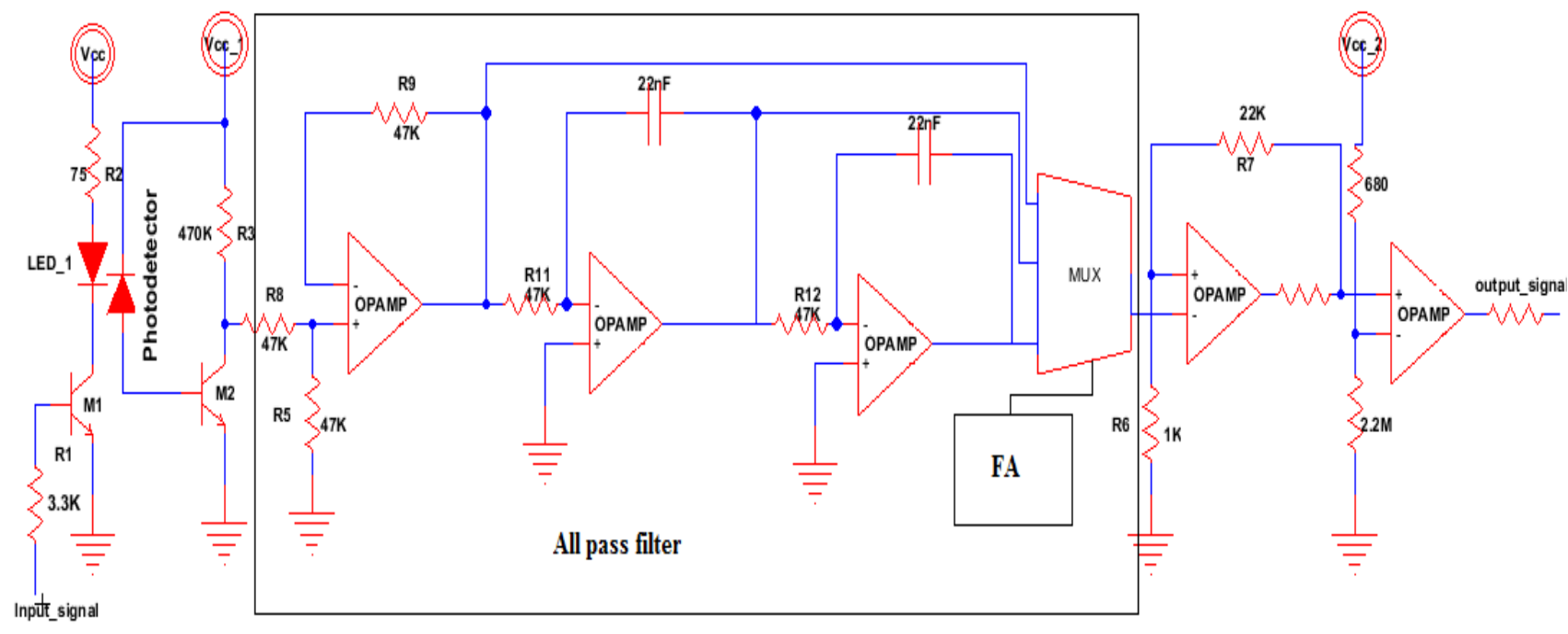

Fig. 2. Equivalent Circuit of VLC system

This equivalent circuit has two different parts such as VLC transmitter and VLC receiver. The components used in the VLC transmitter is LED driver and LED. Moreover, the components used in the VLC receiver are $\mathrm{PD}$, inverting amplifier and voltage comparator. The detailed description of the VLC communication of this proposed system is described as follows:

\section{A. Transmitter}

Initially, the data is converted as binary values and it is written in the .txt file. Then this .txt file is inserted in an electric VLSI to transmit the data through the optical channel. There are various colored LED's used in the VLC such as orange, white, blue, green and red. The LED's used in the VLC are can be purchased at a low price. The red LED used at the transmitter side, due to its higher sensing capacities. The VLC transmitter takes the data in the visible light form. The On-Off Keying (OOK) is used for modulating the LED. The channel model considered among the transmitter and receiver is Line Of Sight (LOS).

\section{B. Channel model}

The visible light (i.e., input) from the LED transmitter is transmitted to the LED receiver through the LOS channel model. There are various advantages acquired by using this LOS channel model such as Inter Symbol Interference (ISI), minimum path loss, dispersion and improved data rate. The transmitted optical power through the LOS model is expressed in Eq. (1). $p_{r}=h(0) p_{i}$

Where, the channel DC gain is represented as $h(0)$ and the transmitted optical power is $p_{i}$.

The noise model is considered in the LOS channel model. In VLC system, there are various noises such as ambient light noise, ambient light-induced shot noise and signal occurred in the photo-diode. Moreover, the thermal noise also occurred in the photodiode. This thermal noise is created by the Trans-Impedance Amplifier (TIA). The receiver LED current is given in the following Eq. (2).

$$
I(t)=\eta p_{i}(t) \otimes h(t)+n(t)
$$

Where, the current of photodetector is $I(t)$; the photosensitivity of the photo detector is $\eta$; the instantaneous input power is $p_{i}(t)$; the impulse response is $h(t)$ and the noise occurred in the optical channel is $n(t)$. The noise function is expressed in the following Eq. (3). $n=\sigma_{\text {ambient }}^{2}$

Where, $n=\sigma_{\text {ambient }}^{2}$ represents the ambient light-induced shot noise that is expressed in the following Eq. (4). The ambient light sources are created from various sources that are incandescent, fluorescent lamps, skylight and sunlight. $n=\sigma_{\text {ambient }}^{2}=2 q R\left(p_{r}+p_{n}\right) B$ 
Where, the electron's charge is $q$; photo diode responsivity is denoted asR $R$; received noise power is represented as $p_{r}$; received optical power is denoted as $p_{n}$ and the receiver bandwidth is $\mathrm{B}$.

\section{Receiver}

The signal affected by the ambient light noise is received by using the PD receiver through the optical channel. The input signal is recovered from the noisy signal by using the Sallen key APF. The detailed description of the Sallen key APF is given in the following section:

\section{a. Sallen Key all pass filter}

The Sallen key APF is used in the proposed system based on the APF butterworth type for attenuating the frequency. This Sallen key APF has simple design and it is selected due to its higher value of quality factor (Q). There are three different filtering processes considered in this proposed system such as low pass, band pass and high pass filters. Thus the spectrum analyzer is used for knowing the characteristics of the artificial lighting and interference for using the filters in VLC. The information from the spectrum analyzer is given to multiplexer (MUX) for identifying which filter output is required to be transmitted to the inverting amplifier. For example, the process of High Pass Filter (HPF) in the Sallen key APF is described as follows:

The equivalent circuit for the Sallen key HPF is shown in Fig. 3.

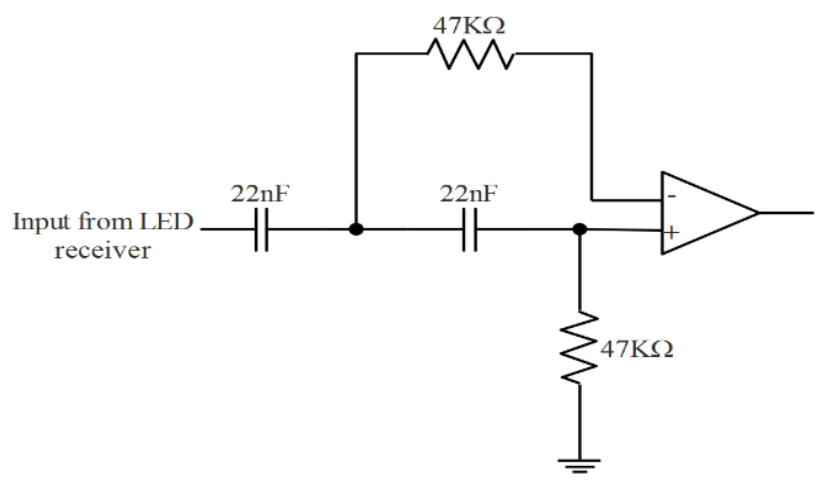

Fig. 3. Equivalent circuit for Sallen key HPF

The cut-off frequency of the Sallen key HPF is expressed in the following Eq. (5).

$$
f_{c}=\frac{1}{2 \pi R C}
$$

The cut off frequency $\left(f_{c}\right)$ considered in this filter design is $150 \mathrm{~Hz}$. For simplifying the filter design, the values of resistors $R_{3}$ and $R_{4}$ are fixed as equal that is $47 \mathrm{~K} \Omega$. Similarly, the capacitor values also fixed as equal that is $22 \mathrm{nF}$. The frequency can't be utilized for occupying the entire information, when the band frequency is greater than the HPF's available bandwidth. So the information about the available bandwidth is required to be known while designing the HPF. The noise from the signal is decreased by using the $150 \mathrm{~Hz}$ cut-off frequency. After finishing the filtering stage, the signal is given as the input to the inverting amplifier.

\section{b. Process of inverting amplifier and voltage comparator}

The inverting amplifier receives the signal from the Sallen key APF for amplifying the received signal. The operational amplifier of inverting amplifier is connected with feedback for generating the closed loop operation. The phase of signal from the Sallen key is changed $180^{\circ}$ out of phase by using the inverting amplifier. The equivalent circuit of the inverting amplifier is shown in Fig. 4.

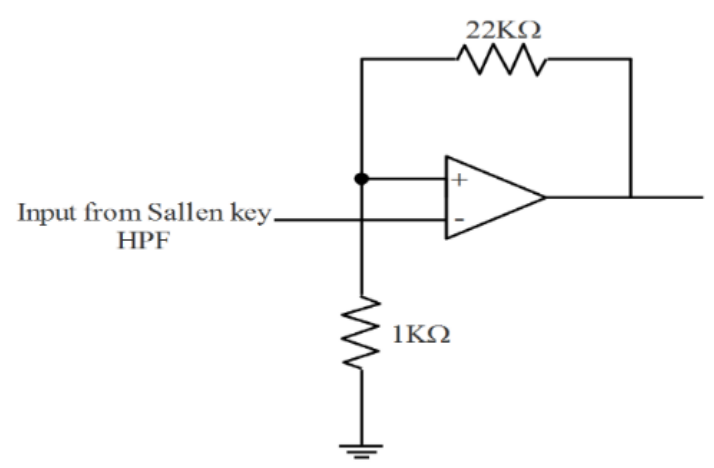

Fig. 4. Equivalent circuit of the inverting amplifier After amplification, the signal is given as the input to the voltage comparator which is shown in Fig. 5. The voltage comparator is used for converting the amplified data signal to digital data signal. The digital signal is obtained by comparing the two different signals one is from the inverting amplifier and other one is reference voltage.

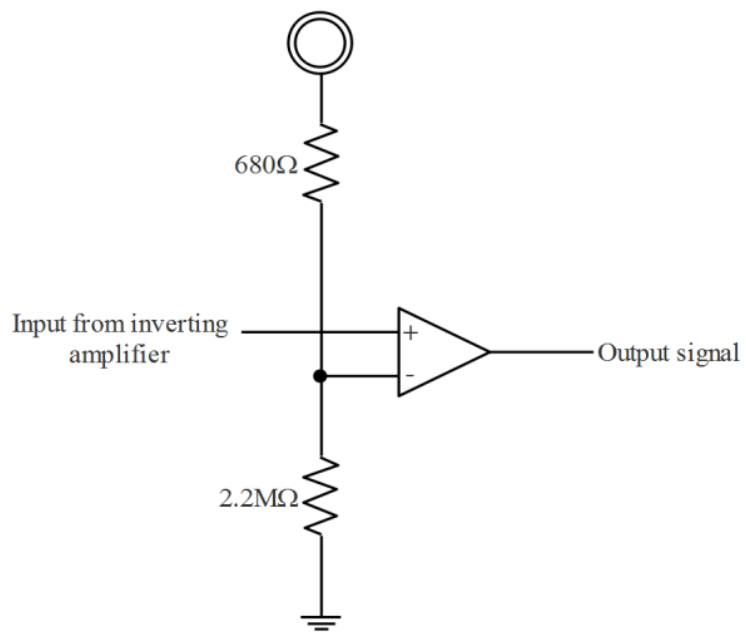

Fig. 5. Equivalent circuit of the voltage comparator

\section{EXPERIMENTAL SETUP}

The VLC of the proposed system is designed in the Electric VLSI software with CMOS 45nm technology. This VLC system uses the Sallen key APF for minimizing the ambient light noise from the data received through the optical channel. The data transmitted through the VLC system is collected by using the MATLAB 2017b 
software in the file extension of .txt. This file has the binary form values of an input signal.

The specifications of red LED used in both the transmitter and receiver of the proposed system is shown in Table 1.

Table I. Specifications of the red LED

\begin{tabular}{|l|l|}
\hline \multicolumn{1}{|c|}{ Parameters } & \multicolumn{1}{c|}{ Value } \\
\hline Model type & King bright WP710A10LSECK/J3 \\
\hline Forward current & $2 \mathrm{~mA}$ \\
\hline luminous intensity & 0.55 Candela[cd] \\
\hline
\end{tabular}

\section{A. Performance measures}

The performance of the proposed methodology has analyzed in terms of area, power consumption, data rate and BER. The descriptions of these parameters are explained as follows:

\section{a. Area}

In the proposed system, the chip area of the VLC system is determined by the logic blocks, interconnections and input/output pads. The size of the circuits may reduce by reducing the switching capacitance and resistance values. The area of the circuit should be less, because a higher area of circuit affects the manufacturing process and also it yields a larger area.

\section{b. Power consumption}

In today's research, low power consumption is the main requirement while designing the hand held devices. So, the system with less power consumption is required to develop modern VLC systems.

\section{c. Data rate}

The VLC systems with a high amount of data rates are required to enhance the performance of the communication system. To enhance the data rate, the noise from the signal is needed to be reduced and also the LED with high amount of luminance capacity is used for performing an effective data transmission.

\section{d. BER}

The BER is defined as the number of errors in a specific unit time. The BER is the ratio of the total number of error bits and the total transmitted bits in a time of interval. The BER is expressed in Eq. (6).

$$
B E R=\frac{\text { Error bitrate }}{\text { Total Transmitted bits }}
$$

\section{RESULTS AND DISCUSSION}

The transmitter of the proposed system transmits the signal by using the red LED through the optical channel and then this transmitted signal converted into optical signal. The input signal transmitted through the optical signal is shown in Fig. 6. Then the ambient light noise is added during the transmission of the signal. The signal with the addition of ambient light noise is shown in Fig. 7. The noise free signal is shown in Fig. 8.

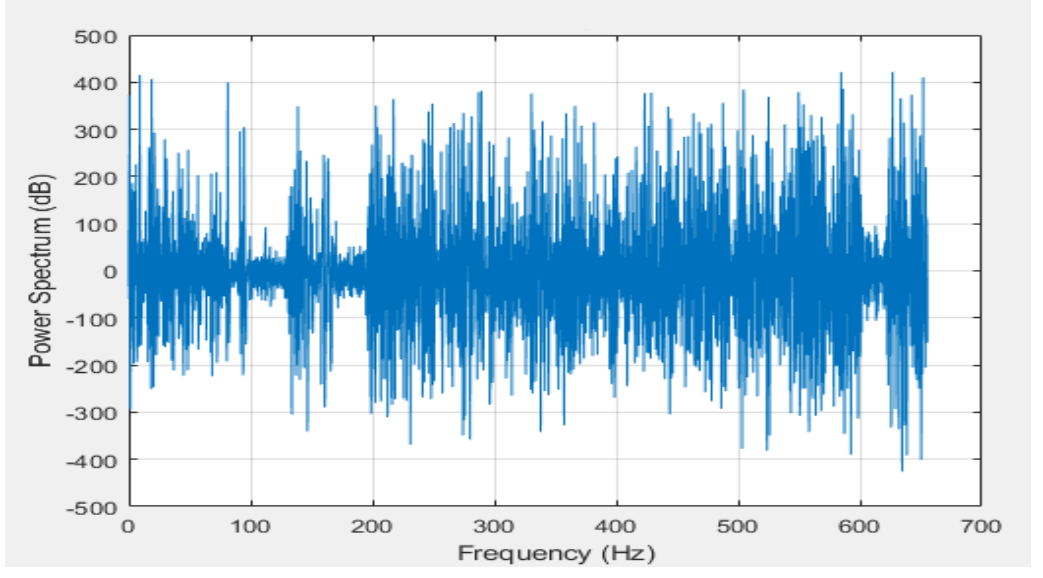

Fig. 6. Input signal

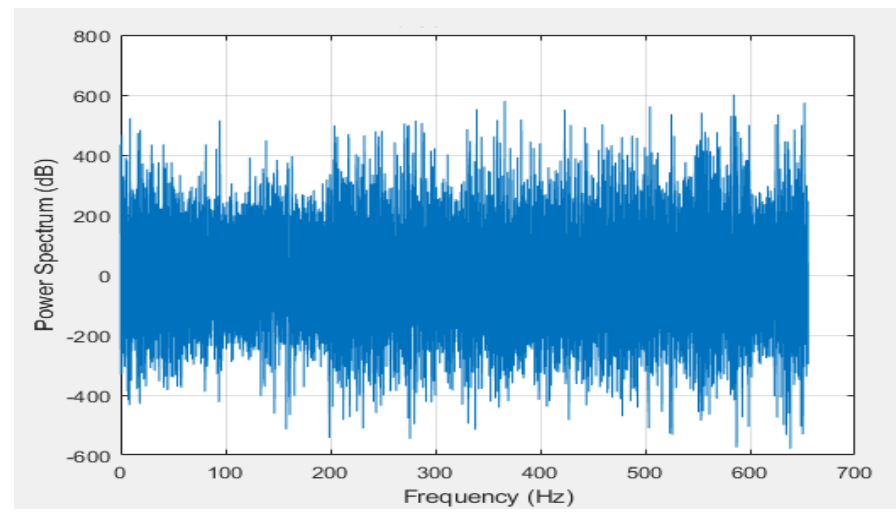

Fig. 7. Received signal 


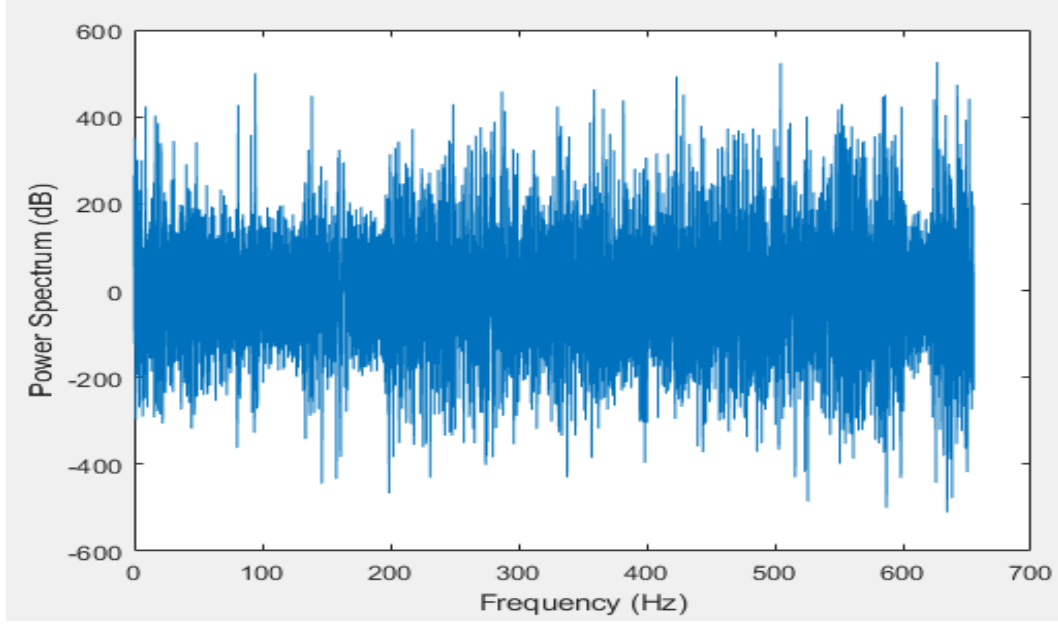

Fig. 8. De-noised output signal

The communication efficiency of the proposed system is without filter. These cases are taken by varying the Signal to mainly evaluated based on BER. This BER has evaluated in Noise Ratio (SNR) and transmission distance in meters (m). two different cases one is the proposed system with filter and This analysis is described as follows:

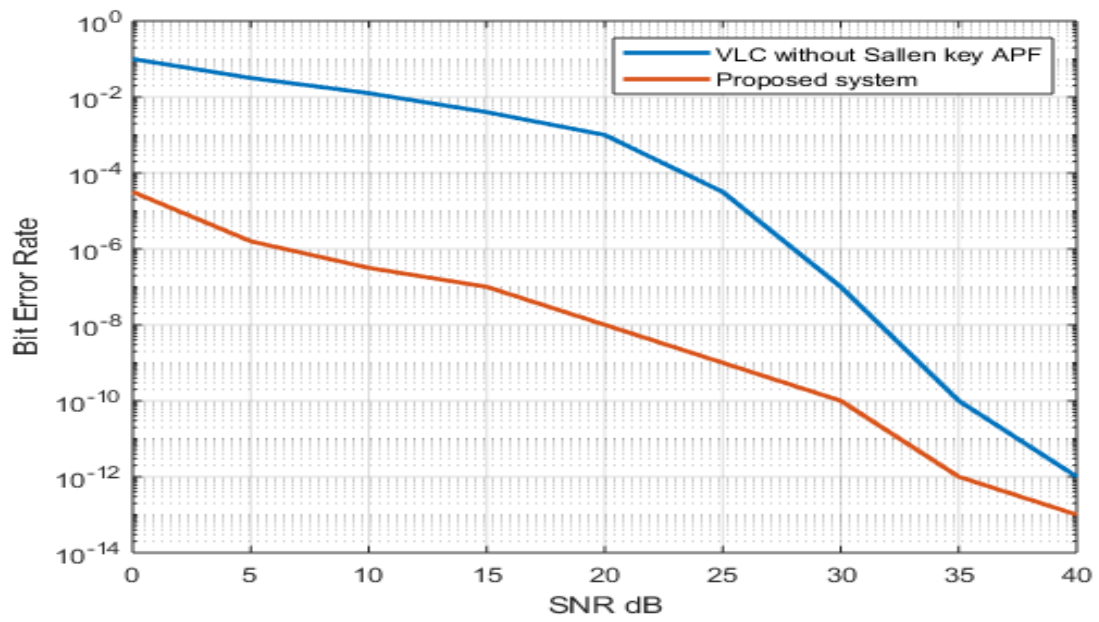

Fig. 8. Performance comparison in terms of SNR vs. BER

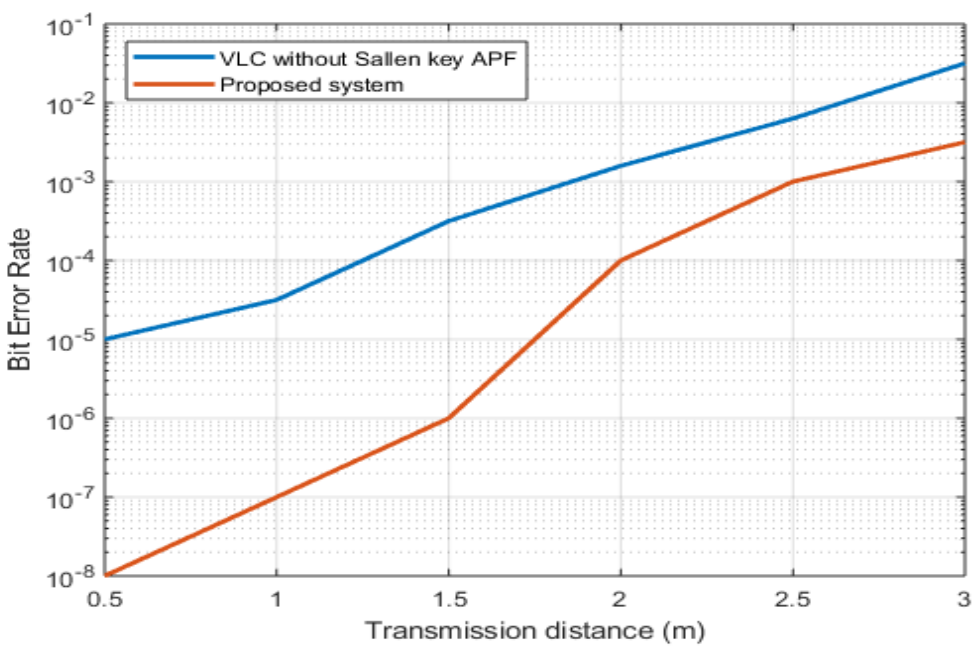

Fig. 9. Performance comparison in terms of Transmission distance vs. BER

Fig. 8 and Fig. 9 shows the BER performance comparison of proposed system with and without Sallen key APF in terms of SNR and transmission distance respectively. From Fig. 7 and Fig. 8 concludes that the proposed system gives better performance when compared to the VLC without any filter.
Because the proposed system has Sallen key APF with cut off frequency to reduce the ambient light noise from the environment. Moreover, to prove the efficiency of the proposed system, it is 
compared with one VLC-LPF [22]. The comparative analysis of the proposed system is given as follows:

Table II. Performance analysis of proposed system

\begin{tabular}{|l|l|l|}
\hline \multicolumn{1}{|c|}{ Parameters } & \multicolumn{1}{|c|}{ VLC-LPF [22] } & \multicolumn{1}{c|}{ Proposed system } \\
\hline Process & $350 \mathrm{~nm}$ technology & $45 \mathrm{~nm}$ technology \\
\hline Area & $4.2 \mathrm{~mm} 2$ & $3.92 \mathrm{~mm} 2$ \\
\hline Modulation scheme & OOK & OOK \\
\hline Power consumption & $8 \mathrm{~W}$ & $6.35 \mathrm{~W}$ \\
\hline Data rate & $8 \mathrm{Mbps}$ & $16 \mathrm{Mbps}$ \\
\hline BER & $<10-6$ at $1 \mathrm{~m}$ & $<10-7$ at $1 \mathrm{~m}$ \\
\hline
\end{tabular}

Table 2 shows the performance analysis of the proposed system with VLC-LPF [22] that contains process technology, area, modulation scheme, power consumption, data rate and BER. From the Table 2 it summarizes that the proposed methodology gives better performance when compared to VLC-LPF [22]. The data rate and BER of the proposed system is increased because of the noise reduction of Sallen key APF with the cut off frequency of $150 \mathrm{~Hz}$ for HPF. This filter removes maximum noise of ambient noise from the received signal.

\section{CONCLUSION}

In this proposed system, the red LED is used at the transmitter due to its high sensing capacity and PD is used at the receiver. The Sallen key APF with an effective cut off frequency is used for reducing the ambient light interference in the VLC system. Because the noise present in the received signal affects the BER of the proposed system. The Sallen key filter has less complexity and also it is inexpensive. The performance of the proposed system is analyzed with VLC system without any filter. In that case, the proposed system gives better BER performance than the VLC system without any filter. Besides, the proposed system is compared with VLC-LPF to know the effectiveness of the proposed system. The performance of the proposed system gives better performance in terms of area, power consumption, data rate and BER when compared to the VLC-LPF. Furthermore, the filter design is optimized by using the advanced technologies like FINFET and CNTFET to design of the filter.

\section{REFERENCES}

1. Y. Yuan, M. Zhang, P. Luo, Z. Ghassemlooy, L. Lang, D. Wang, B. Zhang, and D. Han. (2017) SVM-based detection in visible light communications. Optik, 151, 55-64.

2. M. H. Shoreh, A. Fallahpour, and J. A. Salehi. (2015). Design concepts and performance analysis of multicarrier CDMA for indoor visible light communications. Journal of Optical Communications and Networking, 7(6), pp. 554-562.

3. S. V. Tiwari, A. Sewaiwar, and Y. H. Chung. (2017). Smart home multi-device bidirectional visible light communication. Photonic Network Communications, 33(1), pp. 52-59.

4. C. Gong, S. Li, Q.Gao, and Z. Xu. (2015). Power and rate optimization for visible light communication system with lighting constraints. IEEE transactions on signal processing, 63(16), pp. 4245-4256.

5. S. M. Kim, M. W. Baek, and S. H. Nahm. (2017). Visible light communication using TDMA optical beamforming. EURASIP Journal on Wireless Communications and Networking, 2017(1), pp. 56.

6. A. Khalid, and H. M. Asif. (2018). OCDMA and OSTBC based VLC transceiver design using NI cDAQ. Photonic Network Communications, 35(1), pp. 97-108.

7. D. F. Zhang, Y. J. Zhu, and Y. Y. Zhang. (2013). Multi-LED phase-shifted OOK modulation based visible light communication systems. IEEE Photonics Technology Letters, 25(23), pp. 2251-2254.
8. A. H. Azhar, T.A. Tran, and D. O'Brien. (2012). A gigabit/s indoor wireless transmission using MIMO-OFDM visible-light communications. IEEE photonics technology letters, 25(2), pp. 171-174.

9. G.Pau, M. Collotta, V. Maniscalco, and K. K. R Choo. (2019). A fuzzy-PSO system for indoor localization based on visible light communications. Soft Computing, 23(14), pp.5547-5557.

10. A. M. Căilean, M. Dimian, V. Popa, L. Chassagne, and B. Cagneau, (2016). Novel DSP receiver architecture for multi-channel visible light communications in automotive applications. IEEE Sensors Journal, 16(10), pp. 3597-3602.

11. S. H. Lin, C. Liu, X. Bao, and J. Y. Wang. (2018). Indoor visible light communications: performance evaluation and optimization. EURASIP Journal on Wireless Communications and Networking, 2018(1), pp. 228.

12. Y. Wu, P. Audenaert, M. Pickavet, and D. Colle. Mirror-aided non-LOS VLC channel characterizations with a time-efficient simulation model. Photonic Network Communications, pp.1-16.

13. C. C. Chang, Y. J. Su, U. Kurokawa, and B.I. Choi, (2011). Interference rejection using filter-based sensor array in VLC systems. IEEE sensors journal, 12(5), pp. 1025-1032.

14. H. Farahneh, F. Hussain, and X. Fernando. (2018). Performance analysis of adaptive OFDM modulation scheme in VLC vehicular communication network in realistic noise environment. EURASIP Journal on Wireless Communications and Networking, 2018(1), pp 243.

15. J. H. Yoo, J. S. Jang, J. K. Kwon, H. C. Kim, D.W. Song, and S. Y. Jung, (2016). Demonstration of vehicular visible light communication based on LED headlamp. International journal of automotive technology, 17(2), pp. 347-352.

16. S. Prince, and A. M. Vibin. (2016). Optical Wireless Audio Communication Using LED Lighting System. Wireless Personal Communications, 86(3), pp. 1159-1168.

17. J. Z. Deng, and X. H. Cheng. (2014). An experimental scheme for cordwood color division multiplexing VLC system. Optoelectronics Letters, 10(6), pp. 459-462.

18. F. Che, L. Wu, B. Hussain, X. Li, and C.P. Yue. (2016). A fully integrated IEEE 802.15. 7 visible light communication transmitter with on-chip 8-W 85\% efficiency boost LED driver. Journal of Lightwave Technology, 34(10), pp. 2419-2430.

19. X. Li, B. Hussain, L. Wang, J. Jiang, and C. P. Yue. (2018). Design of a 2.2-mW 24-Mb/s CMOS VLC receiver SoC with ambient light rejection and post-equalization for $\mathrm{Li}-\mathrm{Fi}$ applications. Journal of Lightwave Technology, 36(12), pp. 2366-2375.

20. N. E. Mahfouz, H. A. Fayed, A. A. El Aziz, and M. H. Aly. (2018). Improved light uniformity and SNR employing new LED distribution pattern for indoor applications in VLC system. Optical and Quantum Electronics, 50(9), pp. 350.

21. S. Kadirvelu, and V. Baba. (2018). Visible light communication using LED as receiver with the effect of ambient light. Optical and Quantum Electronics, 50(1), pp. 15.

22. Y. Gao, L. Li, and P. K. Mok. (2018). An AC Input Inductor-Less LED Driver for Efficient Lighting and Visible Light Communication. IEEE Journal of Solid-State Circuits, 53(8), pp. 2343-2355.

\section{Authors ProfiLe}

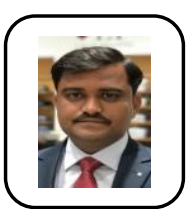

Sharanbasappa Shetkar is pursing Ph.D. under VTU, Belagavi. From 2015. Currently serving as Assistant professor in the Department of Electronics and Communication Engineering at Lingaraj Appa Engineering College from 2012 to till Date.

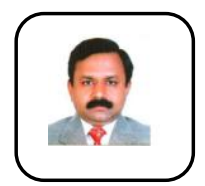

Dr. Baswaraj Gadgay is a Professor and working as Regional Director (I/C) \& PG Coordinator at VTU-RO Kalaburagi. From 2017 to till date. 